\title{
Automated of 74-inch Kottamia Telescope Dome based on Fuzzy Logic Due to Online Weather Station Data
}

\author{
I.M. Selim \\ National Research Institute \\ of Astronomy and \\ Geophysics(NRIAG), Cairo \\ Egypt \\ Higher Technological Institute \\ (HTI),Cairo Egypt
}

\author{
Nasser Ahmed \\ National Research Institute of \\ Astronomy and Geophysics \\ (NRIAG), \\ Cairo Egypt
}

\author{
Maha Lashin \\ Shoubra faculty of \\ Engineering /Banha University, \\ Egypt
}

\begin{abstract}
An overview of automated astronomical facilities (especially in Egypt) is presented. Control of motors speed used to open and close the dome and mirror shutters due to weather station data. The fuzzy logic control introduced as a new control system for the Kottamia astronomical dome's shutters and mirror's shutter due to weather station data. The real operating of Kottamia dome and mirror shutters have been studied to kipping its operation in good performance with weather changes. The weather station data transfer to the fuzzy control system simultaneously. Fuzzy system used to control the opening or closed operation of the dome's shutters and mirror's shutter due to the online homogenous environmental weather station data. In this paper, wind speed, humidity, rain rate, and dust particle are used as an input parameter occurs and the top shutter, bottom shatter and mirror shutter are used as an output parameters reaction.
\end{abstract}

\section{General Terms}

Control systems, Automated Astronomical telescope, weather station data

\section{Keywords}

Fuzzy control systems, Kottamia Astronomical telescope, weather station, DC motor.

\section{INTRODUCTION}

Astronomical observatories are complex technical and scientific for users and purposes. The astronomical community is entering an era of rapid change increasingly characterized by large time domain surveys, robotic telescopes, intelligent system, and automated infrastructures and most significantly of operating modes and scientific consortia that span our individual facilities. Intelligent system is a mechanical system which executes a task with good accuracy and able to adapt itself to changes during the task execution without any kind of human assistance. There is a very wide variety of automated telescope and observatory control software systems operating scientifically useful telescopes with an equally large range of apertures, capabilities, and complexities.

The robotic astronomical observatories able to integrate and coordinate the different automatic subsystems at the observatory, but they require human assistance (teleoperation) for the taking of decisions regarding a given task and/or its supervision. The human assistance in the taking of decisions is replaced by an artificial intelligent system. These are being developed nowadays based on the compilation collected by [1], (see Figure. 1).

The astronomical telescope dome is one of the important item in astronomical observatory. The astronomical telescope dome has some of shutters for the dome and telescope mirror. The astronomical telescope dome not only do they allow you to leave your equipment set up permanently, ready for observing, but also it improves viewing conditions by protecting from the elements and reducing the problem due to the weather conditions.

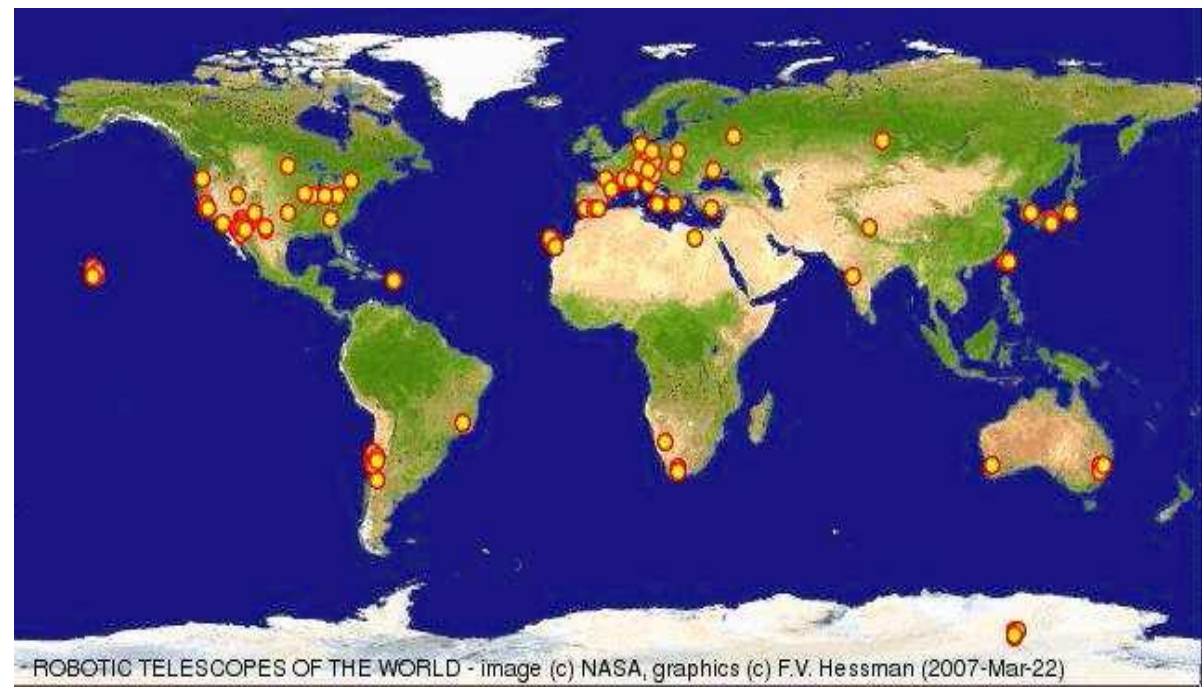

Figure 1: The RAOs location in the world this figures from [1]. 
The weather and the climate are effect in many domains so, measuring of the weather parameters as temperature, humidity, rainfall, wind speed, dew point and atmospherics pressures, etc...; these parameters of the weather can measure by using weather station. The science of the expert system branched from Artificial Intelligence which simulate human thinking who has expertise in the specific domain to solve any problems through knowledge in the expert system [2][3]. Usage of expert system and control system can help us in the work to make decisions which can solve the problem of timing for closing and opening shutters automatic.

The Kottamia dome was constructed by the united Austrian Iron and Steel Works (VOEST) of Linz/Austria. It is $18 \mathrm{~m}$ in diameter with an outer shell of galvanized steel plate painted externally with aluminum as shown in Figure, 2. The dome has two shutters, an upper and a lower capable of moving. Hand operation is possible in case of emergency. The upper shutter is of the "up and over" type whereas the lower shutter is in two parts each opening to the side. At present the dome full controlled with the telescope by ACE system for more details about the dome see [3]. Fuzzy logic is implemented in a standard microcontroller to regulate the speed of a universal motor by a real time adjustment (every 30 millisecond) of the motor current [4]. This microcontroller directly tunes the motor current by means of a chopper converter. It is found that, fuzzy logic approach can be applied to build a closed speed regulation loop from a very low cost tacho-generator. It is applied to fast "real-time" regulation loop without requiring any specific expertise in conventional methods of regulation. The speed of the DC motor controlled by fuzzy logic control based on LABVIEW program [5].

In the present work, fuzzy logic system based on online weather station data has been used to control the motors. The dome and mirror shutters are presented in section 2 . Description and Design Fuzzy Logic Control System presented in section 3. Defining Input and Output of Weather Station are presented in section 4. In section 5 Adjusting fuzzy membership functions and rules, and conclusion has been presented in section 6 .

\section{DOME AND MIRROR SHUTTERS}

The Telescope dome rotated on 26 traveling wheels with 0.17 r.p.m. speed by 6 three phase motors driving 6 pinions into a circular rack. Dome also can be opening of $5 \mathrm{~m}$ wide and extends $2.5 \mathrm{~m}$ beyond the zenith. Other shutters (gates) were lower and the upper one that moving with $4.5 \mathrm{Kw}, 380 \mathrm{~V}$, three gear motors.

Upper shutter is "up and over" type and lower shutter is in two parts each opening to the side, by DC motors as shown in Figure 1. It was a pushbutton for both dome and shutters motions. Now ACE system control is connections between the telescope and the dome [3]. As the telescope tracks the object across the sky, the dome follows the telescope from time to time allow the light of the object enter to the telescope, with computer control of aspects of dome and telescope [3].

The mirror shutter (gate) which consists of twelve steel triangle parts. A1.5 Kw, $380 \mathrm{v}$, and 1350 r.p.m. DC gear motor connected with each part for opening the shutter like flower as shown in Figure 2. [6]

The most common characteristics of the DC motors used for dome shutters, appeared in Table 1.

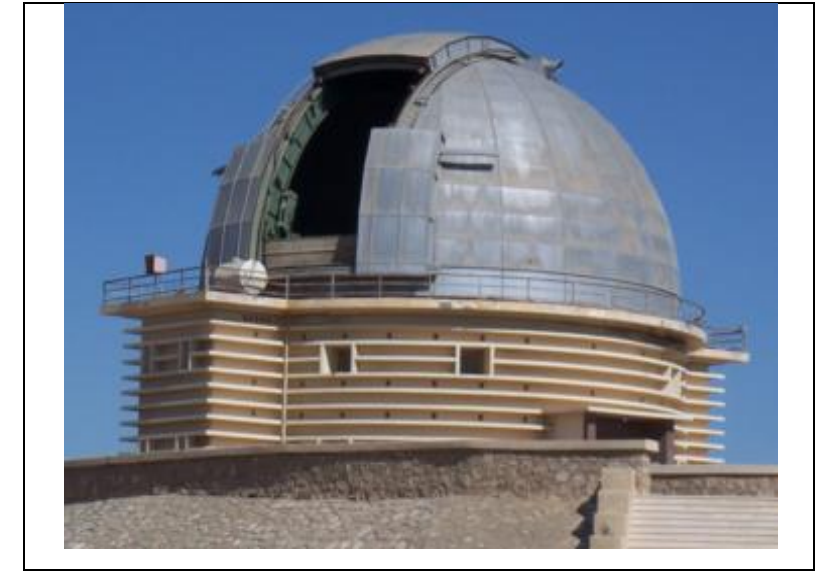

Figure 2: The Kottamia Telescope Dome with open Shutters

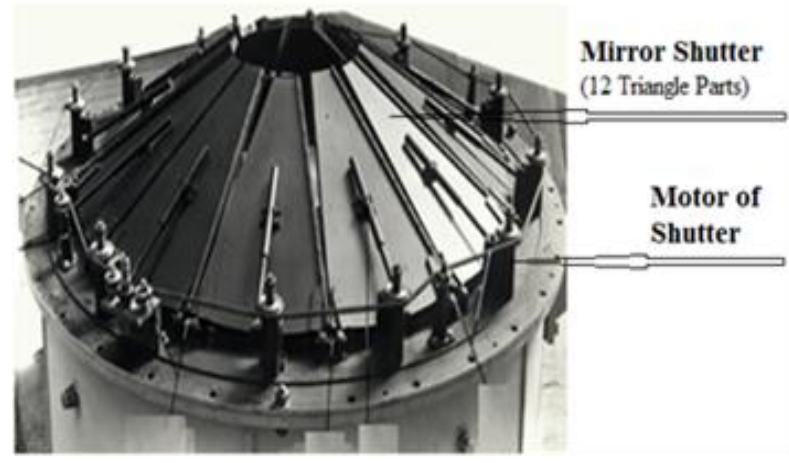

Figure 2: Mirror Shutter

Table 1. DC Three Phase Gear Motor Performance Characteristics

\begin{tabular}{|c||l||l|}
\hline Parameter & \multicolumn{1}{|c|}{$\begin{array}{c}\text { Values Dome } \\
\text { Motor }\end{array}$} & \multicolumn{1}{|c|}{$\begin{array}{c}\text { Values Mirror } \\
\text { Motor }\end{array}$} \\
\hline \hline $\mathrm{R}_{\mathrm{a}}$ & $13.790 \Omega$ & $5.441 \Omega$ \\
\hline $\mathrm{L}_{\mathrm{a}}$ & $0.146 \mathrm{H}$ & $0.0575 \mathrm{H}$ \\
\hline $\mathrm{I}_{\mathrm{a}}$ & $12 \mathrm{~A}$ & $4.734 \mathrm{~A}$ \\
\hline $\mathrm{J}$ & $0.02658 \mathrm{~kg} \cdot \mathrm{m}^{2}$ & $0.01048 \mathrm{~kg} \cdot \mathrm{m}^{2}$ \\
\hline $\mathrm{B}$ & $0.00354 \mathrm{Nms} / \mathrm{rad}$ & $0.0012 \mathrm{Nms} / \mathrm{rad}$ \\
\hline $\mathrm{K}_{\mathrm{b}}$ & $0.54 \mathrm{Vs} / \mathrm{rad}$ & $0.2106 \mathrm{Vs} / \mathrm{rad}$ \\
\hline $\mathrm{K}_{\mathrm{T}}$ & $1.53 \mathrm{Nm} / \mathrm{A}$ & $0.598 \mathrm{Nm} / \mathrm{A}$ \\
\hline
\end{tabular}

\section{DESCRIPTION AND DESIGN FUZZY LOGIC CONTROL SYSTEM}

To protect telescope from the weather changes, it must be control the open or closed of dome and mirror shutters. Fuzzy logic Control (FLC) system is a control system in which a mathematical system that analyzes analog input values in terms of logical variables that take on continuous values between 0 and 1, in contrast to classical or digital logic, which operates on discrete values of either 1 or 0 (true or false) respectively [7][8][9]. Fuzzy Logic provides a simple way to arrive at

a definite conclusion based upon vague, ambiguous, imprecise, noisy, or missing input information. Fuzzy Logic's approach to control problems mimics how a person makes decisions, only much faster.

Components Characteristic of a Fuzzy Controller: 
- $\quad$ Preprocessing

- Fuzzification

- $\quad$ Rule Base

- Defuzzification

- $\quad$ Post processing

Preprocessing: The inputs are most often hard or crisp measurement from some measuring equipment rather than linguistic. A preprocessor block in Figure 5 shows the conditions of measurements before enter the fuzzy controller.

Fuzzification: The first block inside the controller is fuzzification which converts each piece of input data to degrees of membership by a lookup in one or several membership functions.

Rule Base: The collection of rules is called a rule base. The rules are in "If Then "format and formally the If side is called the conditions and the Then side is called the conclusion. The computer is able to execute the rules and compute a control signal depending on the measured inputs error (e) and change in error $(\mathrm{Ce})$.

Defuzzification: is when all the actions that have been activated are combined and converted into a single non-fuzzy output signal which is the control signal of the system. The output levels are depending on the rules that the systems have and the positions depending on the non-linearity's existing to the systems.

Post processing: The post processing block often contains an output gain that can be tuned and also become as an integrator.

\section{DEFINING INPUT AND OUTPUT OF WEATHER STATION}

The weather station used to measure weather parameters like temperature, rain rate, and wind speed, humidity, and dust particles. Several measuring sensors used for this purpose. At a certain level of these parameters the mirror shutter must be closed. On the other side, the rain rate, and humidity levels have the main decision on opening or closed the dome shutter. The range of the measuring values for each parameter's sensor shown in Table 2.

Table 2: Values of Weather Station Sensors Range

\begin{tabular}{|l||l|}
\hline Parameter & Value \\
\hline \hline Temperature & $(-3-50)^{\circ} \mathrm{C}$ \\
\hline Rain rate & $(0.25-50) \mathrm{mm} / \mathrm{hr}$ \\
\hline Wind speed & $(0-15) \mathrm{km} / \mathrm{hr}$ \\
\hline Humidity & $(0-80) \%$ \\
\hline Dust particles & $(0-1.5) \%$ \\
\hline
\end{tabular}

The goal of designed FLC in this study is to open and close the dome and mirror shutters related to weather station values, it done by control the speed of the DC gear motor of this shutters through changing the motor armature voltage value. The cycle of dome and mirror control system can be simplified as shown in Figure 6.

\section{ADJUSTING FUZZY MEMBERSHIP FUNCTIONS AND RULES}

We define the membership function $(m A i)$ for each $(x)$, in the each stage $\mathrm{Si}$ a fuzzy subset fuzzy model as follows:

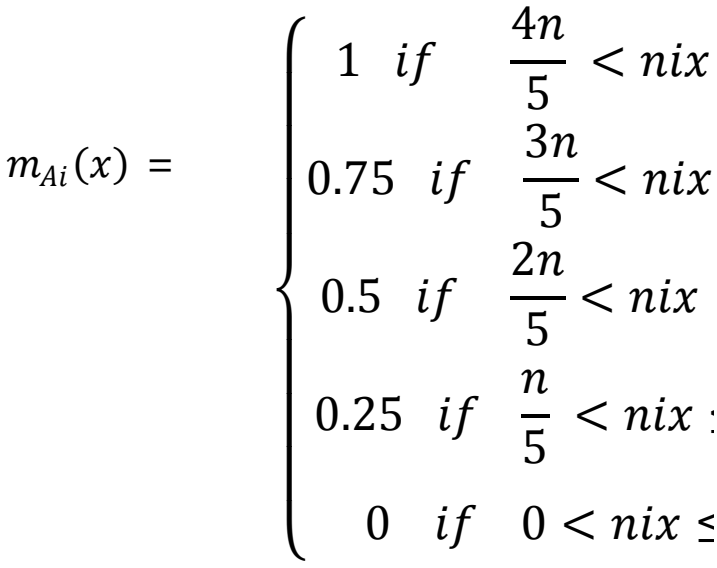

Where the system's $n$ entities (objects), $n \geq 2$, during a process involving vagueness and/or uncertainty. Denote by , $i=1,2,3$. A MATLAB fuzzy logic control system designed to control this operation. The inputs of the fuzzy control system are the output of the weather station. The FLC has 5 inputs, 1 output, and 6 membership functions. The inputs were the output of the

simultaneously weather station (measuring sensors values of temperature, rain rate, wind speed, humidity, and dust particles). The output of the fuzzy system ( 1 or 0$)$ interred to the motor transfer function control equation to control the speed of dome motor (decision of open or closed the shutter). The FLC control system structure shown in Figure 7 with membership functions explained as shown in Figure 8, and the fuzzy system rules shown in Figure 9.

The rule base represented as If-Then statements governing the relationship between the inputs and outputs variables in terms of membership functions. There were 6 rules depending on weather station parameters values as shown in Table 3

\section{CONCLUSION}

Nowadays the automatic control has played a vital role in the advance of engineering and science. The control of direct current (DC) motor is a common practice thus the implementation of DC motor of controller speed is important. In this paper fuzzy logic control have been used to control the speed of a DC motor of Kottamia Astronomical Dome and mirror shutters to open and close the dome and mirror shutters based on simultaneously weather station data, wind speed, humidity, rain rate, temperature, and dust particle. The proposed fuzzy Logic controller for control the Kottamia astronomical dome has more advantages, such as higher flexibility, control, better dynamic and static performance. The results fuzzy logic response shows that the overshoot, settling time, peak time and control performance are greatly by using a Fuzzy Logic Controller. The simulation results are obtained using MATLAB/SIMULINK. Hence, Fuzzy logic controller design was proposed and implemented. 


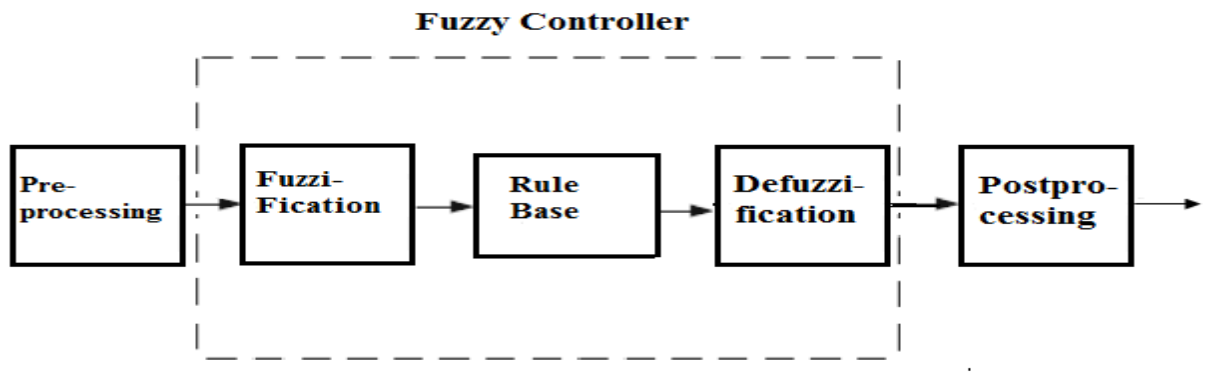

Figure 5: Process Blocks for a Fuzzy Controller

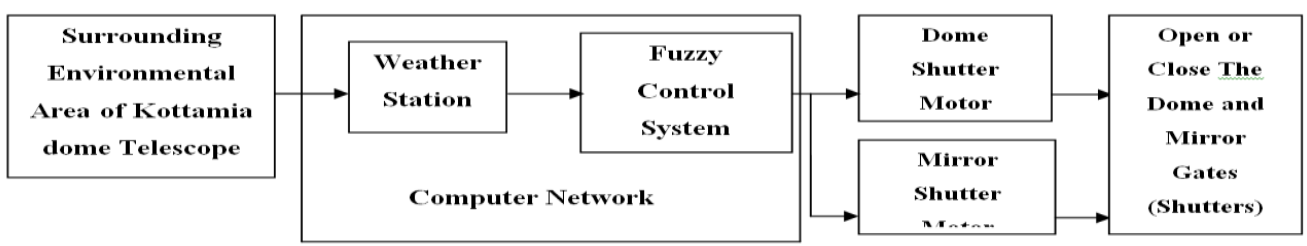

Figure 6: Scheme of Control System Used

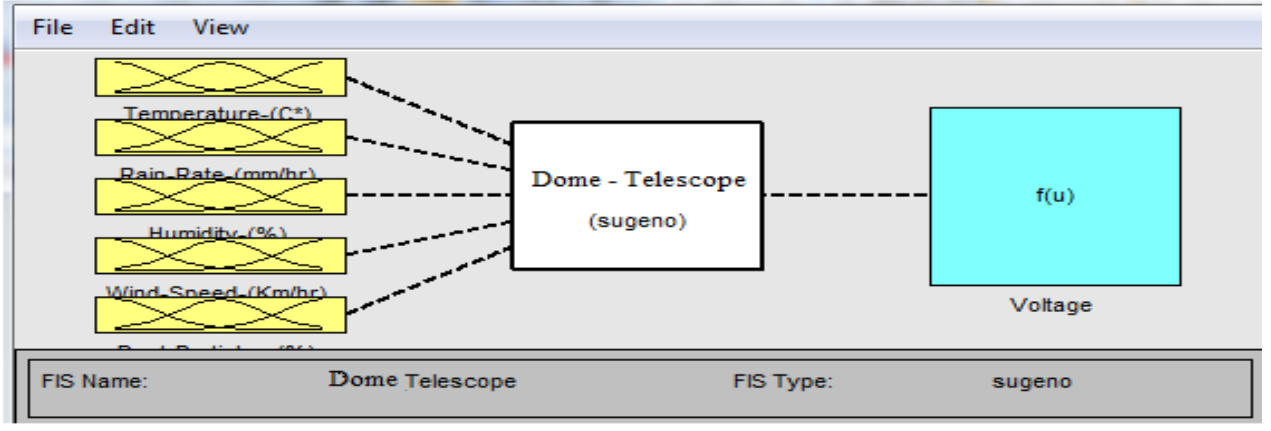

Figure 7: FLC Control System Structure

Table 3: Rule Base Parameters

\begin{tabular}{|c|c|c|c|c||c||c|}
\hline \hline Parameters & Temperature & Rain rate & $\begin{array}{c}\text { Wind } \\
\text { speed }\end{array}$ & Humidity & $\begin{array}{c}\text { Dust } \\
\text { particles }\end{array}$ & $\begin{array}{c}\text { Output } \\
\text { value }\end{array}$ \\
\hline \hline Case 1 & Low & Low & Low & Low & Low & 1 \\
\hline Case 2 & High & Low & High & Low & Low & 1 \\
\hline Case 3 & High & High & High & High & High & 0 \\
\hline Case 4 & Low & High & Low & Low & Low & 0 \\
\hline Case 5 & Low & Low & Low & Low & High & 0 \\
\hline Case 6 & Low & Low & Low & High & Low & 0 \\
\hline
\end{tabular}

a
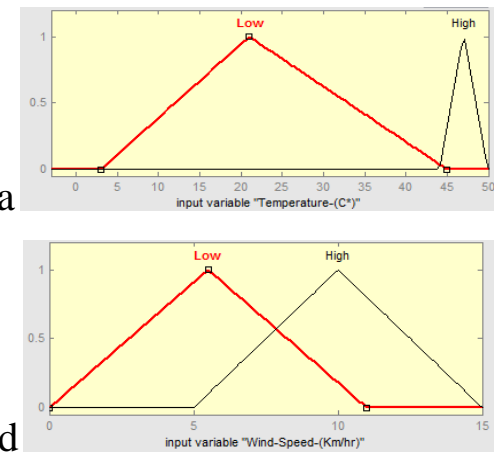

b

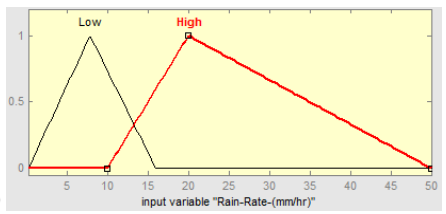

e

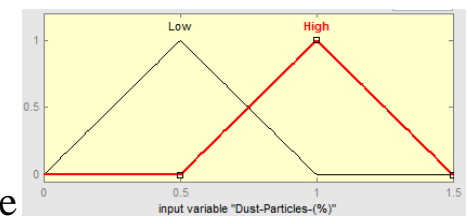

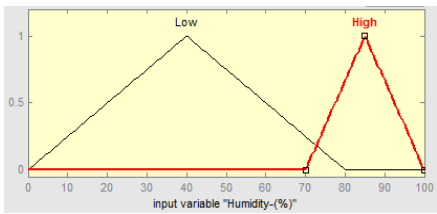$$
\text { . }
$$

\footnotetext{
Figure 8: Membership Functions for (a) Temperature, (b) Rain Rate, (c) Humidity, (d) Wind Speed, and (e) Dust Particles
} 


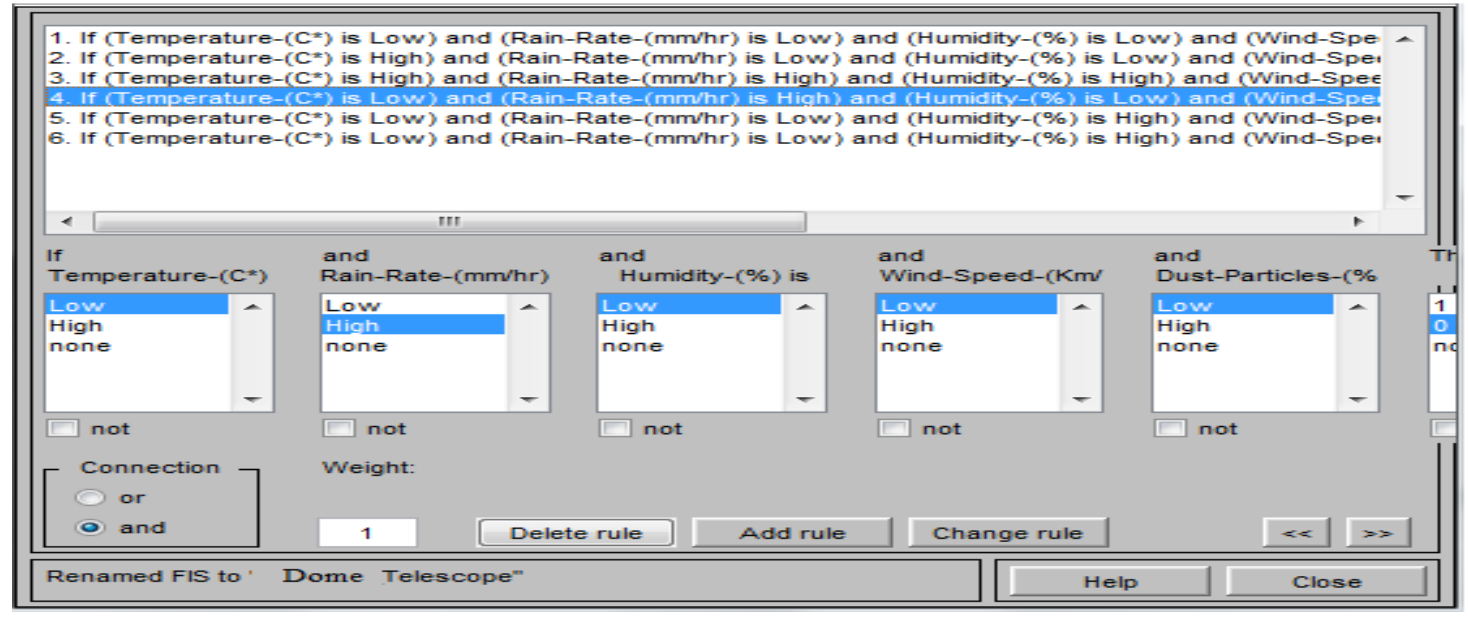

Figure 9: FLC Control System Rules Editor

\section{REFERENCES}

[1] ALBERTO J. CASTRO-TIRADO LNEA III, 2008. A.Ulla \& M. Manteiga (editors).

[2] Azzam, Y. A., Ali, G. B., Ismail, H. A., Haroon, Selim, I. "Workshop on the International Heliophysical Basic Space Science", UN/ESA/NASA, 2009.

[3] Fengler, Wolfgang, Ahya Ihsan, and Kai Kaiser. Managing Post-Disaster Reconstruction Finance. Vol. 4475. World Bank Publications, 2008.

[4] Pierre Guillemin, "Fuzzy Logic Applied to Motor Control", IEEE Transactions on Industry Applications, Vol. 32, NO. 1, 1996.

[5] Salim, Jyoti Ohri, and Naveen," Speed Control of DC Motor using Fuzzy Logic based on LabVIEW", International Journal of Scientific and Research Publications, Vol. 3, 2013.
[6] Guoshing Huang, Shuocheng Lee, "PC-based PID Speed Control in DC Motor", Department of Electronic Engineering, National Chin-Yi University of Technology.

[7] Husain Ahmed, " Controlling of D.C. Motor using Fuzzy Logic Controller", Conference on Advances in Communication and Control Systems, 2013.

[8] Umesh Kumar Bansal, and Rakesh Narvey," Speed Control of DC Motor Using Fuzzy PID Controller", Advance in Electronic and Electric Engineering, Vol. 3, pp.1209-1220, 2013.

[9] Klir, J., George, Yuan, Bo. "Fuzzy Sets and Fuzzy Logic Theory and Applications", Prentice Hall PTR,1995. 